\title{
Immagini religiose nel mondo romano. Nove saggi
}

\section{Claudia Beltrão e Federico Santangelo}

\section{(2) OpenEdition}

\section{Journals}

\section{Edizione digitale}

URL: https://journals.openedition.org/mythos/3818

DOI: $10.4000 /$ mythos.3818

ISSN: 2037-7746

\section{Editore}

Salvatore Sciascia Editore

\section{Notizia bibliografica digitale}

Claudia Beltrão e Federico Santangelo, «Immagini religiose nel mondo romano. Nove saggi», Mythos

[Online], 15 | 2021, online dal 15 décembre 2021, consultato il 15 décembre 2021. URL: http:// journals.openedition.org/mythos/3818; DOI: https://doi.org/10.4000/mythos.3818

Questo documento è stato generato automaticamente il 15 décembre 2021.

Mythos 


\title{
Immagini religiose nel mondo romano. Nove saggi
}

\author{
Claudia Beltrão e Federico Santangelo
}

1 Qual è il ruolo delle immagini nelle dinamiche religiose, e come occorre valutarne l'importanza? Creare l'immagine di una divinità o renderla visibile ha un impatto sulla prassi, sulla devozione religiosa e sulla più vasta riflessione che le accompagna e le sostiene; conferisce inoltre all'essere divino un grado di presenza nella dimensione materiale. Il linguaggio delle immagini, gli elementi iconografici, le concezioni spaziali e l'uso stesso dello spazio creano modalità di visibilità, di prossimità o di distanza degli dèi. Le forme di comportamento, le azioni, le performances che creano o presentano il divino, e i testi che ne configurano l'immaginazione e la comprensione sono attestati da diversi tipi di fonti, in varie situazioni e in vari contesti. Quando pensiamo all'immagine divina, l'aspetto materiale e quello visivo convergono, si mescolano e si attuano a vicenda. D'altra parte, operare alcune distinzioni analitiche è il passo necessario che ci permette di parlare delle immagini. I diversi approcci disciplinari e metodologici, a loro volta, mettono in gioco questioni complesse: le strategie di legittimazione, gli attori religiosi (compresi gli artisti e i committenti) e i diversi usi delle immagini (cultuali, teologici, politici).

2 Tutti questi elementi esprimono e comunicano messaggi, sia in contesti religiosi propriamente detti (templi, santuari), che in altri ambiti. La ricerca intorno alle immagini religiose non si propone soltanto di comprendere i codici e decrittarli attraverso i metodi dell'analisi iconografica o semiologica. Nonostante essa rappresenti un primo momento fondamentale, non ci si può fermare a quel livello, poiché i codici sono continuamente riformulati nei loro significati attraverso tempi e contesti diversi, e una figura religiosa attestata nel lungo periodo può vedersi conferiti significati nuovi in quadri mutati. Vari studi recenti hanno permesso di focalizzare con maggior precisione il significato delle evidenze materiali nello studio delle religioni, mettendo alla prova e perfezionando metodologie che traggono beneficio dal dialogo interdisciplinare. Osservare la materialità della religione, d'altra parte, non fa che rendere più pressanti gli interrogativi intorno al divino, all'antropomorfismo, alle 
modalità di identificazione della divinità e ai diversi generi entro cui essa si può classificare. Soprattutto, mette in risalto la dimensione estetica della religione e il suo carattere specialistico, ponendo le pratiche cultuali e la presenza delle divinità in luoghi specifici.

Il cosiddetto visual turn ha proposto una critica al ruolo privilegiato delle analisi testuali nello studio delle religioni, dando risalto all'uso religioso delle immagini anche nella letteratura e nei testi sacri. Al tempo stesso, le strategie per conferire presenza e accessibilità al divino, e la sua dipendenza da discorsi verbali che configurano l'immaginazione e il pensiero dei fedeli, talvolta per limitare o rendere illecite alcune forme dell'uso delle immagini, sono temi che sollecitano ricerche nuove. Lo studio dell'interazione fra immagini, immaginazione e immaginario nei testi, e l'analisi della rappresentazione verbale e visiva del divino portano nuovi contributi alla comprensione delle percezioni della cultura visiva in diverse religioni e società. Si può, almeno provvisoriamente, sostenere che alcune delle questioni metodologiche più importanti gravitino intorno alla costruzione dell'idea di divinità, alla sua attuazione, $\mathrm{e}$ a come diversi dialoghi abbiano luogo entro quel processo. Le singole discipline si misurano con le immagini divine in modi differenti, attraverso tradizioni metodologiche distinte e diverse agende di ricerca, così come attraverso le varie forme di sistematizzazione teorica e concettuale. Da qui la crescente rilevanza del dibattito sul complesso tema delle immagini divine. In un'immensa mole di documenti, la statua antropomorfica non è che una fra i vari elementi e i vari modi di misurarsi con il divino, di rendere la sua presenza visibile e percettibile, di facilitare la comunicazione fra esseri umani e divinità. Le divinità erano e sono visibili non soltanto attraverso oggetti materiali, ma anche in altre forme e modalità: ad esempio, gli attori umani (il sacerdote, gli officianti), coloro che hanno una funzione direttiva nei rituali e nei loro riferimenti al divino, e i politici e gli intellettuali, che danno senso, significato e configurano diverse modalità di vedere e percepire il divino. I nove contributi raccolti in questo volume tentano di esplorare le forme attraverso cui si è tentato di immaginare la presenza e l'azione degli dèi, le modalità attraverso cui le immagini divine sono create e in cui le iconografie e gli elementi visivi vengono appropriati, così come l'evocazione di immagini specifiche nella mente umana, percorrendo un arco temporale e geografico che include l'esperienza storica del mondo romano nella sua ampiezza.

4 I primi cinque contributi di questa raccolta concentrano l'attenzione sulla città di Roma. I primi tre si misurano con temi che pertengono al rapporto fra religione, immagine e spazio. Il contributo di Giorgio Ferri, The Place-Making Function of Ritual Movement at Rome: From the Salians to Our Lady of Mount Carmel, pone il tema del movimento rituale, e in particolare delle processioni, nella costruzione dei luoghi e del paesaggio sacro romano in due casi di studio, uno antico e uno moderno. Tracciare $\mathrm{i}$ movimenti rituali significa cercare di comprenderne il carattere performativo, le emozioni e i sentimenti di appartenenza religiosa e comunitaria che essi suscitano e, in particolare, la creazione di quello che Ferri definisce, richiamandosi a un recente sviluppo storiografico, lived ritual space. A sua volta, Guillermina Bogdan, in El dinamismo de las imágenes sagradas: análisis de las figuras de Marte, Término, Juventas y Júpiter en Antiquitates rerum divinarum 40-41, de Varrón, discute gli intenti e le ambizioni di un testo antiquario in quanto fonte per lo studio di un illustre spazio sacro nella città di Roma: il tempio di Giove Capitolino. Il testo di Varrone si impone come una guida alla comprensione delle divinità e degli spazi sacri, e come una metodologia per la 
costruzione di tali figure divine, che incide sulla formazione dell'identità e della memoria romane, tanto per i contemporanei come per i posteri. Il contributo di Cecilia Ames, Religious Images and Urban Places in the Roman World in Vergil's Aeneid, pone invece il tema dei mutamenti conosciuti dallo spazio urbano a Roma nel periodo augusteo, quando diversi luoghi della città furono riappropriati e trasformati dall'iniziativa di diversi agenti e da nuove pratiche religiose. Focalizzando l'attenzione sul libro VIII dell'Eneide, Ames dimostra come il poeta collochi i momenti della sua narrazione nella città di Roma, ponendo l'accento su manifestazioni rituali, immagini religiose e luoghi di memoria, e mettendo in rapporto lo spazio religioso e lo spazio urbano.

5 Il saggio di Claudia Beltrão, Cicerone e le statue degli dèi, si misura con tre diverse modalità attraverso le quali Cicerone mira a evocare specifiche immagini riguardanti gli dèi nelle menti dei suoi ascoltatori e dei suoi lettori. L'accento viene posto sul carattere persuasivo dell'uso delle immagini divine da parte del grande oratore e pensatore romano. Ogni esperienza religiosa è ancorata in una narrazione culturale più ampia, che può essere anche ricodificata per conferire visibilità al divino, sia in linea con esperienze culturali tradizionali che nel quadro di contro-narrazioni, e l'articolo di María Emilia Cairo, Iuno Regina in Aeneid 12: Evocatio and Reconciliation, discute l'uso poetico del rituale della evocatio da parte di Virgilio nel dialogo fra Giove e Giunone nel libro XII dell'Eneide. In questo dialogo Cairo riconosce un'allusione a distinti momenti nei quali l'esistenza stessa di Roma era minacciata e, in particolare, all'evocatio di Giunone dopo la presa di Veio nel 396 a.C. Giunone appare nel poema come la divinità italica che difende gli elementi italici nella costruzione della futura identità romana.

Gli altri quattro articoli allargano il quadro geografico della discussione e si misurano con immagini religiose situate oltre la città di Roma e l'Italia, attraverso un amplissimo arco temporale. Gilvan Ventura da Silva, in Images of the Feast in Antioch: Some Reflections about Dionysus' Mosaics in Domestic Settings, ci conduce nella vibrante Antiochia tardoimperiale, con le sue variegate celebrazioni religiose, il suo ethos festivo e le sue longeve forme di culto dionisiaco. Ventura discute le immagini musive del dio e le personalità divine associate agli dei che frequentano i triclinia antiocheni e i loro rapporti con il simposio. Considerazioni di carattere materiale, quali le dimensioni delle statue e le opzioni spaziali per l'interazione con l'immagine divina, così come i testi epigrafici che l'affiancano e la integrano, sono decisive per la comprensione di queste immagini. Nello stesso spirito, secondo Carlos Machado (Secular and Sacred in Late Roman Portraits) il tema della concettualizzazione dei contesti deve essere posto al centro dell'analisi, superando la radicata opposizione che differenzia gli oggetti e gli spazi sacri rispetto a quelli profani, e sostituendola con un quadro di rapporti molteplici, che coinvolgono attori umani, elementi materiali, livelli di contesto, assetti spaziali, valorizzando la plasticità e la polisemia delle immagini stesse.

7 Nicole Belayche, in Coping with Images of Initiations in the Mithras Cult, discute invece il tema delle immagini associate, con vari gradi di plausibilità, ai culti misterici e, in particolare, al culto di Mitra entro diversi contesti architettonici, e non soltanto in luoghi di culto esplicitamente definiti come tali: un tema che continua a suscitare intensi dibattiti. Belayche discute problemi di importanza centrale: i princìpi generali attraverso cui si possa identificare l'immagine della scena di un mistero; la natura intrinsecamente polisemica di un fenomeno religioso come i «misteri»; il ruolo delle immagini di iniziazione nel culto mitraico. Le immagini si affermano come documenti che creano i propri significati: costruzioni visive che delineano una narrazione 
intellettuale, speculativa o immaginaria sulla realtà, che risulta dunque plasmata anche dall'ambiente culturale in cui prende forma. La raccolta si conclude con l'articolo di Jorwan Costa Junior, Immagini religiose del mondo romano nella letteratura scolastica del Brasile contemporaneo, nel quale si esplora un importante e sinora trascurato risvolto dell'attualità delle immagini religiose nel mondo romano. Attraverso lo studio di un problema di ricezione dell'antico, Costa Junior dimostra come i libri scolastici attualmente in uso in Brasile e approvati dalle autorità pubbliche rappresentino il cristianesimo come l'unica religione propriamente detta dell'impero romano, riducendo al minimo o rimuovendo del tutto l'influenza di ogni altra religione del Mediterraneo antico. Vengono poi discussi gli effetti di questa narrazione sul pubblico destinatario di quei volumi - i bambini e gli adolescenti che frequentano le scuole pubbliche in Brasile. Secondo Costa Junior, l'aggregato di narrazioni e di immagini religiose proposto nei libri di testo afferma l'inesorabilità del cristianesimo, non solo in rapporto ad altre religioni antiche, ma anche alle religioni attualmente praticate nel Brasile moderno, specialmente quelle di matrice africana. Le immagini e le forme di pensiero e di espressione del divino sono tuttora uno dei grandi temi di dibattito e di conflitto dell'esperienza.

8 Le società umane riformulano costantemente la loro riflessione sul divino. La natura ambigua - o polisemica - delle divinità è un terreno di ricerca affascinante e ancora aperto a numerose linee di indagine. Questa raccolta di saggi non ha certo pretese di esaustività, ma ambisce a offrire un contributo collettivo al dibattito sulle immagini religiose nel mondo romano. I lavori presentati qui traggono origine da un convegno tenutosi alla Universidade Federal do Estado do Rio de Janeiro (UNIRIO) dal 16 al 18 luglio 2019, con l'intento di promuovere un confronto fra un ampio campo di approcci, e di riunire storici dell'antichità, storici dell'arte, classicisti, storici della filosofia antica, ed esperti della ricezione del mondo antico, allargando il confronto oltre $\mathrm{i}$ termini consueti della storia delle religioni. Siamo grati alla British Academy, che ha reso possibile il convegno nel quadro di una Newton Advanced Fellowship, e alla Newcastle University e a UNIRIO per il supporto istituzionale che ci hanno accordato; a Elizabeth Bell, Jorwan Costa Junior e Patricia Horvat per il sostegno all'organizzazione del convegno; ai colleghi che hanno aderito a questo progetto; e al pubblico che ha preso parte alle tre giornate del colloquio, contribuendo attivamente al nostro dibattito.

9 Abbiamo infine uno speciale debito di riconoscenza verso Daniela Bonanno, Corinne Bonnet e gli altri componenti della Direzione di Mythos per la generosità con cui hanno voluto considerare questa raccolta di saggi, e verso i revisori anonimi della Rivista, dalle cui osservazioni abbiamo tratto enorme profitto. 


\section{AUTORI}

\section{CLAUDIA BELTRÃO}

Departamento de História

Universidade Federal do Estado do Rio de Janeiro - UNIRIO

Av. Pasteur, 458

Urca - Rio de Janeiro - RJ

CEP 22290-255

Brasil

claudia.rosa(at)unirio.br

\section{FEDERICO SANTANGELO}

School of History, Classics and Archaeology

Armstrong Building

Newcastle University

Newcastle upon Tyne NE1 7RU

United Kingdom

federico.santangelo(at)newcastle.ac.uk 